\title{
Linear Precoding Scheme Design for MIMO Two-Way Relay Systems with Imperfect Channel State Information
}

\author{
Xiao-min Chen, Jun-xu Su, Qiu-ming Zhu, Xu-jun Hu, and Zhu Fang \\ College of Electronic and Information Engineering, Nanjing University of Aeronautics and Astronautics, Nanjing, China \\ Correspondence should be addressed to Xiao-min Chen; chenxm402@nuaa.edu.cn
}

Received 16 November 2016; Revised 16 February 2017; Accepted 1 March 2017; Published 12 April 2017

Academic Editor: Nazrul Islam

Copyright (c) 2017 Xiao-min Chen et al. This is an open access article distributed under the Creative Commons Attribution License, which permits unrestricted use, distribution, and reproduction in any medium, provided the original work is properly cited.

\begin{abstract}
The aim of this paper is to investigate a linear precoding scheme design for a multiple-input multiple-output two-way relay system with imperfect channel state information. The scheme design is simplified as an optimal problem with precoding matrix variables, which is deduced with the maximum power constraint at the relay station based on the minimum mean square error criterion. With channel feedback delay at both ends of the channel and the channel estimation errors being taken into account, we propose a matrix decomposition scheme and a joint iterative scheme to minimize the average sum mean square error. The matrix decomposition method is used to derive the closed form of the relay matrix, and the joint iterative algorithm is used to optimize the precoding matrix and the processing matrix. According to numerical simulation results, the matrix decomposition scheme reduces the system bit error rate (BER) effectively and the joint iterative scheme achieves the best performance of BER against existing methods.
\end{abstract}

\section{Introduction}

Multiple-input multiple-output (MIMO) technology has become the key technology owing to its ability to increase system capacity and improve spectral efficiency without increasing the bandwidth [1]. The relay technology is a common space diversity method that can effectively reduce the effects of channel fading. The combination of MIMO with relay technology can further improve the capacity and system performance of the relay network. There has been extensive theoretical research on the performance improvement of unidirectional MIMO relay system [2, 3]. In fact, the MIMO two-way relay system has received great deal of attention recently owing to its high spectrum efficiency $[4,5]$.

Some existing studies have investigated for MIMO relay systems. In the actual communication system, the reliability of the communication link could be ensured by minimizing the mean square error (MSE) of the receiver. In references $[6,7]$ analysis under the condition of perfect channel state information (CSI), the precoding algorithm regards the MSE at two source nodes as the optimization target; the study [8] presents the complete CSI and the joint optimization method based on the criterion of MSE duality of two-way MIMO relay system. In [9], the precoding algorithm of amplifyand-forward MIMO two-way relay system based on channel capacity analysis is studied under limited channel feedback condition. However, the precoding scheme only considered the power constraints of the relay node without considering the power constraints of the transmitter. The paper [10] details the design of the combined precoding scheme for the two-way relay system, using the iterative method to optimize the combined precoding matrix. However, due to the time-varying wireless channel noise, the existence of the desired CSI is not realistic. References [11-13] researched for the amplify-and-forward two-way MIMO relay system, assuming that, in the relay terminal known source-relay, relay destination channel from the channel estimation errors and transmit antenna correlation proposed a joint transceiver design scheme based on the imperfect CSI. The study [14] presents the precoding algorithm with consideration channel feedback delay and channel estimation errors; however, it is only suitable for the MIMO system.

Although some excellent works about precoding design have been done for MIMO two-way relay networks [1517], most of them have not taken the channel estimation errors and feedback delay into account. However, in practical 


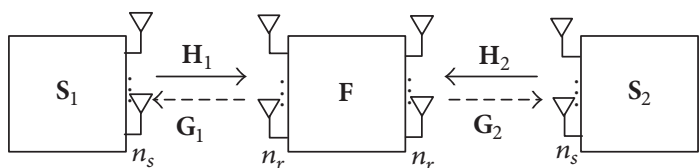

FIGURE 1: Diagram of MIMO two-way relay system.

networks, due to the limitation of the channel estimation method and the existence of channel feedback delay, the ideal CSI is difficult to obtain. This motivates us to investigate the problem of optimal precoding design in MIMO twoway relay networks in the presence of channel estimation errors and feedback delay. In this paper, a MIMO two-way relay system is considered where the channel estimation error and feedback delay between relay node and the destination exist. At the relay node, the linear precoding design method is produced based on the minimum mean square error (MMSE) rule. Finally, the optimal precoding matrix and the destination linear matrix are derived using the theoretical proof.

\section{System Model and Channel Model}

2.1. System Model. Considering MIMO two-way relay system as shown in Figure 1, which consists of two source nodes $S_{1}$ and $S_{2}$ with one relay node denoted by $\mathbf{F}$, both $S_{1}$ and $S_{2}$ are equipped with $n_{s}$ antennas. Relay node $\mathbf{F}$ has $n_{r}$ antennas. At present, the research on the performance of MIMO twoway relay system is based on the model of time division duplex (TDD). During the first phase, source nodes $S_{1}$ and $S_{2}$ transmit their information to relay node $\mathbf{F}$. In the second time slot, relay node multiplies its received signal by a linear precoding matrix $\mathbf{F} \in \mathbb{C}^{N_{r} \times N_{r}}$ and forwards it to $S_{1}$ and $S_{2}$, respectively. It is assumed that all nodes are operating in half-duplex mode due to the hardware complexity and the relay node transmits and receives in two orthogonal time slots.

In the first time slot, source nodes $S_{i}(i=1,2)$ transmit information $\mathbf{x}_{i} \in \mathbb{C}^{n_{s} \times 1}$ to relay node, $\mathbf{x}_{i}$ is satisfied with covariance matrix $\mathbf{R}_{x_{i}}=\varepsilon\left[\mathbf{x}_{i} \mathbf{x}_{i}^{H}\right], \varepsilon[\bullet]$ donates the statistical expectation, and then the signal vector $\mathbf{y}_{s} \in \mathbb{C}^{n_{r} \times 1}$ received at relay node is

$$
\mathbf{y}_{s}=\mathbf{H}_{1} \mathbf{x}_{1}+\mathbf{H}_{2} \mathbf{x}_{2}+\mathbf{n}_{r} .
$$

Here $\mathbf{H}_{i} \in \mathbb{C}^{n_{r} \times n_{s}}$ represents the channel matrix of $S_{i}$ to $\mathbf{F}$ and $\mathbf{n}_{r} \in \mathbb{C}^{n_{r} \times 1}$ denotes the additive white Gaussian noise (AWGN) vector with covariance matrix $\mathbf{R}_{n_{r}}=\varepsilon\left[\mathbf{n}_{r} \mathbf{n}_{r}^{H}\right]$.

In the second time slot, relay node multiplies its received signal and forwards it to $S_{1}$ and $S_{2}$, respectively. The received signal $\mathbf{y}_{i} \in \mathbb{C}^{n_{s} \times 1}$ at $S_{i}$ can be expressed as

$$
\mathbf{y}_{i}=\mathbf{H}_{i}^{H} \mathbf{F H}_{j} \mathbf{x}_{j}+\mathbf{H}_{i}^{H} \mathbf{F} \mathbf{H}_{i} \mathbf{x}_{i}+\mathbf{H}_{i}^{H} \mathbf{F} \mathbf{n}_{r}+\mathbf{n}_{i},
$$

where $j=2$ if $i=1$ and $j=1$ if $i=2 . \mathbf{n}_{i} \in \mathbb{C}^{n_{s} \times 1}$ denotes the AWGN vector with covariance matrix $\mathbf{R}_{n_{i}}=\varepsilon\left[\mathbf{n}_{i} \mathbf{n}_{i}^{H}\right]$.
2.2. Channel Model. Considering the channel estimation error and channel feedback delay, the channel matrix at time instant $t$ can be expressed as [18, 19]

$$
\mathbf{H}_{t}=\widehat{\mathbf{H}}_{t}+\mathbf{T}_{t}+\mathbf{\Xi}_{t}
$$

where $\boldsymbol{\Xi}_{t}$ denotes the channel estimation error matrix and $\mathbf{T}_{t}$ is the delay estimation error matrix. With a certain amount of estimation error the channel matrix $\mathbf{H}_{t}$ changes to be $\widehat{\mathbf{H}}_{t}$. In addition, after experiencing a period of time $\tau$ for feedback delay, $\widehat{\mathbf{H}}_{t}$ turns to be $\sqrt{\rho} \widehat{\mathbf{H}}_{t-\tau}$. Then the channel matrix $\mathbf{H}_{t}$ can be written as [19]

$$
\mathbf{H}_{t}=\sqrt{\rho} \widehat{\mathbf{H}}_{t-\tau}+\mathbf{T}_{t}+\boldsymbol{\Xi}_{t}
$$

where $\rho$ is the channel correlation coefficient formulated as in [19]. For Clarke's fading spectrum, $\rho$ could be obtained as $\rho=$ $J_{0}^{2}\left(2 \pi f_{d} \tau\right)$, and here $J_{0}(\bullet)$ is the zeroth-order Bessel function of the first kind and $f_{d}$ is the Doppler frequency [20].

In this paper, we discuss the channel matrix of sourcerelay and relay-source at a certain time, and correlation coefficient $\rho$ is related to the delay coefficient $\tau$. For the convenience of expression, $\mathbf{H}_{t, i}$ and $\widehat{\mathbf{H}}_{t-\tau, i}$ could be written as $\mathbf{H}_{t}$ and $\widehat{\mathbf{H}}_{i}$. Therefore, our channel matrix could be further expressed as [21]

$$
\mathbf{H}_{i}=\sqrt{\rho_{i}} \widehat{\mathbf{H}}_{i}+\mathbf{T}_{i}+\boldsymbol{\Xi}_{i}
$$

$\boldsymbol{\Xi}_{t}$ is independent of $\mathbf{T}_{i}$ and its elements are subjected to $\mathrm{CN}\left(0, \sigma_{e}^{2}\right) . \mathrm{CN}\left(u, \sigma^{2}\right)$ represents the complex Gauss distribution with a mean value of $u$ and a variance of $\sigma^{2}$. Let $\Sigma_{i}=$ $\mathbf{T}_{i}+\boldsymbol{\Xi}_{t}$ and $\mathbf{H}_{t}$ can be noted as

$$
\mathbf{H}_{i}=\sqrt{\rho_{i}} \overline{\mathbf{H}}_{i}+\Sigma_{i} .
$$

Each element of $\Sigma_{i}$ is subjected to $\mathrm{CN}\left(0,\left(1-\rho_{i}^{2}\right)\left(1-\sigma_{e_{i}}^{2}\right)+\sigma_{e_{i}}^{2}\right)$. Let $\sigma_{\Sigma_{i}}^{2}=\left(1-\rho_{i}^{2}\right)\left(1-\sigma_{e_{i}}^{2}\right)+\sigma_{e_{i}}^{2}$. The receiver signal $\widetilde{\mathbf{y}}_{i}$ at $S_{i}$ is

$$
\widetilde{\mathbf{y}}_{i}=\mathbf{y}_{i}-\rho_{i} \overline{\mathbf{H}}_{i}^{H} \mathbf{F} \overline{\mathbf{H}}_{i} \mathbf{x}_{i}=\mathbf{H}_{i}^{H} \mathbf{F} \mathbf{H}_{j} \mathbf{x}_{j}+\mathbf{H}_{i}^{H} \mathbf{F} \mathbf{n}_{r}+\mathbf{n}_{i}+\mathbf{k}_{i},
$$

where $\mathbf{k}_{i}=\left(\mathbf{H}_{i}^{H} \mathbf{F} \mathbf{H}_{i}-\rho_{i} \overline{\mathbf{H}}_{i}^{H} \mathbf{F} \overline{\mathbf{H}}_{i}\right) \mathbf{x}_{i}$ is the residual self-interference (SI) with covariance matrix $\mathbf{R}_{k_{i}}=\varepsilon\left[\mathbf{k}_{i} \mathbf{k}_{i}^{H}\right]$. When the detection matrix $\mathbf{Q}_{i}$ is employed by $S_{i}$, the mean squared error (MSE) of the detected signal at $S_{i}$ can be expressed as below:

$$
\operatorname{MSE}_{i}\left(\mathbf{F}, \mathbf{Q}_{i}\right)=\varepsilon\left(\left\|\mathbf{Q}_{i} \widetilde{\mathbf{y}}_{i}-\mathbf{x}_{j}\right\|^{2}\right) .
$$

\section{Problem Formulation}

In this paper, a joint design of the relay precoding matrix $\mathbf{F}$ and the detection matrixes $\mathbf{Q}_{1}$ and $\mathbf{Q}_{2}$ is proposed with the maximum transmission power constraint based on the MMSE criterion [22]. Taking the channel feedback delay and 
estimation errors into account, the MSE expression of $S_{i}$ can be further represented as [23]

$$
\begin{aligned}
\operatorname{MSE}_{i} & \left(\mathbf{F}, \mathbf{Q}_{i}\right)=\operatorname{Tr}\left\{\varepsilon_{\boldsymbol{\Sigma}_{i}}\left[\left\|\mathbf{Q}_{i} \widetilde{\mathbf{y}}_{i}-\mathbf{x}_{j}\right\|^{2}\right]\right\} \\
= & \operatorname{Tr}\left\{\varepsilon_{\boldsymbol{\Sigma}_{1}, \boldsymbol{\Sigma}_{2}}\left[\left(\mathbf{Q}_{i} \mathbf{H}_{i}^{H} \mathbf{F} \mathbf{H}_{j}\right) \mathbf{R}_{x_{j}}\left(\mathbf{Q}_{i} \mathbf{H}_{i}^{H} \mathbf{F} \mathbf{H}_{j}\right)^{H}\right]\right\} \\
& +\operatorname{Tr}\left[\varepsilon_{\boldsymbol{\Sigma}_{i}}\left(\mathbf{Q}_{i} \mathbf{H}_{i}^{H} \mathbf{F} \mathbf{R}_{n_{r}} \mathbf{F}^{H} \mathbf{H}_{i} \mathbf{Q}_{i}^{H}\right)\right] \\
& -\operatorname{Tr}\left\{2 \operatorname{Re}\left[\varepsilon_{\mathbf{\Sigma}_{1}, \boldsymbol{\Sigma}_{2}}\left(\mathbf{Q}_{i} \mathbf{H}_{i}^{H} \mathbf{F} \mathbf{H}_{j} \mathbf{R}_{x_{i}}\right)\right]\right\} \\
& +\operatorname{Tr}\left[\varepsilon_{\boldsymbol{\Sigma}_{i}}\left(\mathbf{Q}_{i} \mathbf{R}_{k_{i}} \mathbf{Q}_{i}^{H}\right)\right]+\operatorname{Tr}\left(\mathbf{R}_{x_{j}}\right) \\
& +\operatorname{Tr}\left(\mathbf{Q}_{i} \mathbf{R}_{n_{i}} \mathbf{Q}_{i}^{H}\right)
\end{aligned}
$$

where $\operatorname{Tr}[\bullet]$ is the trace of the matrix and $\operatorname{Re}[\bullet]$ denotes the real part. It is assumed that each of the elements in a random matrix $\mathbf{x} \in \mathbb{C}^{n \times n}$ is an independent and identically distributed zero mean complex Gauss random variable, $\mathbf{x}$ is satisfied with $\operatorname{Tr}\left(\mathbf{x} \mathbf{A} \mathbf{x}^{H}\right)=\sigma_{x}^{2} \mathbf{I}_{n}$, and here $\mathbf{I}_{n}$ is $n \times n$ unit matrix. We assume the variance of $\boldsymbol{\Sigma}_{i}$ is much smaller than one. Hence, we have

$$
\begin{aligned}
\operatorname{Tr} & {\left[\varepsilon_{\boldsymbol{\Sigma}_{i}}\left(\mathbf{Q}_{i} \mathbf{R}_{k_{i}} \mathbf{Q}_{i}^{H}\right)\right] } \\
& =\rho_{i} \operatorname{Tr}\left\{\varepsilon _ { \boldsymbol { \Sigma } _ { i } } \left[\mathbf { Q } _ { i } \left(\overline{\mathbf{H}}_{i}^{H} \mathbf{F} \boldsymbol{\Sigma}_{i} \mathbf{R}_{x_{i}} \boldsymbol{\Sigma}_{i}^{H} \mathbf{F}^{H} \overline{\mathbf{H}}_{i}\right.\right.\right. \\
& +\boldsymbol{\Sigma}_{i}^{H} \mathbf{F} \overline{\mathbf{H}}_{i} \mathbf{R}_{x_{i}} \overline{\mathbf{H}}_{i}^{H} \mathbf{F}^{H} \boldsymbol{\Sigma}_{i}+\overline{\mathbf{H}}_{i}^{H} \mathbf{F} \boldsymbol{\Sigma}_{i} \mathbf{R}_{x_{i}} \overline{\mathbf{H}}_{i}^{H} \mathbf{F}^{H} \boldsymbol{\Sigma}_{i} \\
& \left.\left.\left.+\overline{\mathbf{H}}_{i}^{H} \mathbf{F} \boldsymbol{\Sigma}_{i} \mathbf{R}_{x_{i}} \overline{\mathbf{H}}_{i}^{H} \mathbf{F}^{H} \boldsymbol{\Sigma}\right) \mathbf{Q}_{i}^{H}\right]\right\} \\
& =\rho_{i} \operatorname{Tr}\left[\mathbf{Q}_{i} \overline{\mathbf{H}}_{i}^{H} \mathbf{F} \mathbf{Z}_{i} \mathbf{F}^{H} \overline{\mathbf{H}}_{i} \mathbf{Q}_{i}^{H} \operatorname{Tr}\left(\mathbf{R}_{x_{i}}\right)\right. \\
& \left.+\operatorname{Tr}\left(\mathbf{F} \overline{\mathbf{H}}_{i} \mathbf{R}_{x_{i}} \overline{\mathbf{H}}_{i}^{H} \mathbf{F}^{H}\right) \mathbf{Q}_{i} \mathbf{Z}_{i} \mathbf{Q}_{i}^{H}\right] .
\end{aligned}
$$

Here $\mathbf{Z}_{i}=\sigma_{\Sigma_{i}}^{2} \mathbf{I}_{n_{s}}$. In the same way, some simplifications can be written as

$$
\begin{aligned}
\operatorname{Tr} & \left\{\varepsilon_{\boldsymbol{\Sigma}_{1}, \boldsymbol{\Sigma}_{2}}\left[\left(\mathbf{Q}_{i} \mathbf{H}_{i}^{H} \mathbf{F} \mathbf{H}_{j}\right) \mathbf{R}_{x_{j}}\left(\mathbf{Q}_{i} \mathbf{H}_{i}^{H} \mathbf{F} \mathbf{H}_{j}\right)^{H}\right]\right\} \\
& =\rho_{i} \rho_{j} \operatorname{Tr}\left(\mathbf{Q}_{i} \overline{\mathbf{H}}_{i}^{H} \mathbf{F H}_{j} \mathbf{R}_{x_{j}} \overline{\mathbf{H}}_{j}^{H} \mathbf{F}^{H} \overline{\mathbf{H}}_{i} \mathbf{Q}_{i}^{H}\right) \\
& +\rho_{i} \operatorname{Tr}\left[\mathbf{Q}_{i} \overline{\mathbf{H}}_{i}^{H} \mathbf{F} \mathbf{Z}_{i} \mathbf{F}^{H} \overline{\mathbf{H}}_{i} \mathbf{Q}_{i}^{H} \operatorname{Tr}\left(\mathbf{R}_{x_{i}}\right)\right] \\
& +\rho_{j} \operatorname{Tr}\left[\mathbf{Q}_{i} \mathbf{Q}_{i}^{H} \operatorname{Tr}\left(\mathbf{F} \overline{\mathbf{H}}_{j} \mathbf{R}_{x_{j}} \overline{\mathbf{H}}_{j}^{H} \mathbf{F}^{H} \mathbf{Z}_{i}\right)\right] \\
& \left.+\rho_{i} \operatorname{Tr}\left\{\mathbf{Q}_{i} \overline{\mathbf{H}}_{i}^{H} \overline{\mathbf{H}}_{i} \mathbf{Q}_{i}^{H} \operatorname{Tr}_{[\mathbf{F}} \mathbf{Z}_{i} \mathbf{F}^{H} \operatorname{Tr}\left(\mathbf{R}_{x_{i}}\right)\right]\right\} \\
& =\rho_{i} \operatorname{Tr}\left(\mathbf{Q}_{i} \overline{\mathbf{H}}_{i}^{H} \mathbf{F} \boldsymbol{\Pi}_{j} \mathbf{F}^{H} \overline{\mathbf{H}}_{i} \mathbf{Q}_{i}^{H}\right) \\
& +\operatorname{Tr}\left[\mathbf{Q}_{i} \mathbf{Z}_{i} \mathbf{Q}_{i}^{H} \operatorname{Tr}\left(\mathbf{F} \boldsymbol{H}_{j} \mathbf{F}^{H}\right)\right],
\end{aligned}
$$

$$
\begin{aligned}
\operatorname{Tr} & {\left[\varepsilon_{\boldsymbol{\Sigma}_{i}}\left(\mathbf{Q}_{i} \mathbf{H}_{i}^{H} \mathbf{F R}_{n_{r}} \mathbf{F}^{H} \mathbf{H}_{i} \mathbf{Q}_{i}^{H}\right)\right] } \\
= & \operatorname{Tr}\left\{\varepsilon _ { \boldsymbol { \Sigma } _ { i } } \left[\mathbf{Q}_{i}\left(\sqrt{\rho_{i}} \overline{\mathbf{H}}_{i}^{H}+\boldsymbol{\Sigma}_{i}^{H}\right)\right.\right. \\
\cdot & \left.\left.\mathbf{F R}_{n_{r}} \mathbf{F}^{H}\left(\sqrt{\rho_{i}} \overline{\mathbf{H}}_{i}+\boldsymbol{\Sigma}_{i}\right) \mathbf{Q}_{i}^{H}\right]\right\} \\
= & \rho_{i} \operatorname{Tr}\left[\mathbf{Q}_{i} \overline{\mathbf{H}}_{i}^{H} \mathbf{F R}_{n_{r}} \mathbf{F}^{H} \overline{\mathbf{H}}_{i} \mathbf{Q}_{i}^{H}\right] \\
+ & \operatorname{Tr}\left[\mathbf{Q}_{i} \mathbf{Z}_{i} \mathbf{Q}_{i}^{H} \operatorname{Tr}\left(\mathbf{F R}_{n_{r}} \mathbf{F}^{H}\right)\right] \\
\operatorname{Tr} & \left\{2 \operatorname{Re}\left[\varepsilon_{\boldsymbol{\Sigma}_{1}, \boldsymbol{\Sigma}_{2}}\left(\mathbf{Q}_{i} \mathbf{H}_{i}^{H} \mathbf{F H}_{j} \mathbf{R}_{x_{i}}\right)\right]\right\} \\
= & 2 \sqrt{\rho_{i} \rho_{j}} \operatorname{Tr}\left[\operatorname{Re}\left(\mathbf{Q}_{i} \overline{\mathbf{H}}_{i}^{H} \mathbf{F} \overline{\mathbf{H}}_{j} \mathbf{R}_{x_{i}}\right)\right],
\end{aligned}
$$

where $\boldsymbol{\Pi}_{j}=\overline{\mathbf{H}}_{j} \mathbf{R}_{x_{j}} \overline{\mathbf{H}}_{j}^{H}+\operatorname{Tr}\left(\mathbf{R}_{x_{j}}\right) \mathbf{Z}_{i}$; substituting (10) (11) into (9), the original expression (9) can be simplified as

$$
\begin{aligned}
\operatorname{MSE}_{i}\left(\mathbf{F}, \mathbf{Q}_{i}\right)= & \rho_{i} \operatorname{Tr}\left(\mathbf{Q}_{i} \overline{\mathbf{H}}_{i}^{H} \mathbf{F} \boldsymbol{\Theta}_{i} \mathbf{F}^{H} \overline{\mathbf{H}}_{i} \mathbf{Q}_{i}^{H}\right)+\operatorname{Tr}\left(\mathbf{R}_{s_{j}}\right) \\
& +\operatorname{Tr}\left(\mathbf{Q}_{i} \mathbf{\Phi}_{i} \mathbf{Q}_{i}^{H}\right) \\
& -2 \sqrt{\rho_{i} \rho_{j}} \operatorname{Re}\left[\operatorname{Tr}\left(\mathbf{Q}_{i} \overline{\mathbf{H}}_{i}^{H} \mathbf{F} \overline{\mathbf{H}}_{j} \mathbf{R}_{x_{i}}\right)\right],
\end{aligned}
$$

where

$$
\begin{aligned}
& \boldsymbol{\Theta}_{i}=\boldsymbol{\Pi}_{j}+\mathbf{R}_{n_{r}}+\operatorname{Tr}\left(\mathbf{R}_{x_{i}}\right) \mathbf{Z}_{i}, \\
& \boldsymbol{\Phi}_{i}=\operatorname{Tr}\left[\mathbf{F}\left(\boldsymbol{\Pi}_{j}+\mathbf{R}_{n_{r}}+\rho_{i} \overline{\mathbf{H}}_{i} \mathbf{R}_{x_{i}} \overline{\mathbf{H}}_{i}^{H}\right) \mathbf{F}^{H} \mathbf{Z}_{i}+\mathbf{R}_{n_{i}}\right] .
\end{aligned}
$$

We are now ready to design detection matrix $\mathbf{Q}_{i}$ and precoding matrix $\mathbf{F}$ to minimize the average sum MSE of $S_{i}$. The problem can be formulated as

$$
\begin{array}{cl}
\min _{\mathbf{Q}_{1}, \mathbf{Q}_{2}, \mathbf{F}} & \operatorname{MSE}=\operatorname{MSE}_{1}\left(\mathbf{F}, \mathbf{Q}_{1}\right)+\operatorname{MSE}_{2}\left(\mathbf{F}, \mathbf{Q}_{2}\right) \\
\text { s.t. } & \operatorname{Tr}\left(\mathbf{F R}_{r} \mathbf{F}^{H}\right) \leq P_{r}
\end{array}
$$

and here

$$
\mathbf{R}_{r}=\varepsilon_{\boldsymbol{\Sigma}_{i}}\left[\mathbf{y}_{s} \mathbf{y}_{s}^{H}\right]=\Pi_{1}+\Pi_{2}+\mathbf{R}_{n_{r}} .
$$

\section{The MMSE-Based Joint Precoding Scheme Design}

4.1. Matrix Decomposition Algorithm Design. Since the joint iterative algorithm is being proposed, this paper introduces a kind of matrix decomposition algorithm with relatively low computational complexity. For (12), when the precoding matrix $\mathbf{F}$ is determined, the optimal linear detection matrix $\mathbf{Q}_{i}$ can be uniquely determined.

From the following condition $\left.(\partial \mathrm{MSE} / \partial \mathbf{Q})\right|_{\mathbf{Q}_{i}=\mathbf{Q}_{i}^{*}}=0, \mathbf{Q}_{i}$ can be written as

$$
\mathbf{Q}_{i}=\rho_{j} \mathbf{R}_{x_{i}} \overline{\mathbf{H}}_{j}^{H} \mathbf{F}^{H} \overline{\mathbf{H}}_{j}\left(\rho_{i} \overline{\mathbf{H}}_{i}^{H} \mathbf{F} \boldsymbol{\Theta}_{i} \mathbf{F}^{H} \overline{\mathbf{H}}_{i}+\boldsymbol{\Phi}_{i}\right)^{-1} .
$$


Substituting (16) into (12), then (12) can be expressed as

$$
\begin{aligned}
& \operatorname{MSE}_{i}\left(\mathbf{F}, \mathbf{Q}_{i}\right)=\operatorname{Tr}\left(\mathbf{R}_{x_{j}}\right) \\
& -\rho_{i} \rho_{j} \operatorname{Tr}\left[\mathbf{R}_{x_{j}} \overline{\mathbf{H}}_{j}^{H} \mathbf{F}^{H} \overline{\mathbf{H}}_{i}\left(\rho_{i} \overline{\mathbf{H}}_{i}^{H} \mathbf{F} \boldsymbol{\Theta}_{i} \mathbf{F}^{H} \overline{\mathbf{H}}_{i}+\mathbf{\Phi}_{i}\right)^{-1}\right. \\
& \left.\cdot \overline{\mathbf{H}}_{i}^{H} \mathbf{F} \overline{\mathbf{H}}_{j} \mathbf{R}_{x_{j}}\right]=\operatorname{Tr}\left(\mathbf{R}_{x_{j}}\right) \\
& -\rho_{j} \operatorname{Tr}\left(\mathbf{R}_{x_{j}} \overline{\mathbf{H}}_{j}^{H} \boldsymbol{\Theta}_{i}^{-1} \overline{\mathbf{H}}_{j} \mathbf{R}_{x_{j}}\right)+p_{i}(\mathbf{F}),
\end{aligned}
$$

where

$$
\begin{aligned}
& p_{i}(\mathbf{F}) \\
& \quad=\rho_{j} \operatorname{Tr}\left[\mathbf { R } _ { x _ { j } } \overline { \mathbf { H } } _ { j } ^ { H } \boldsymbol { \Theta } _ { i } ^ { - 1 / 2 } \left(\rho_{i} \mathbf{Q}_{i}^{1 / 2} \mathbf{F}^{H} \overline{\mathbf{H}}_{i} \boldsymbol{\Phi}_{i}^{-1} \overline{\mathbf{H}}_{i}^{H} \mathbf{F} \mathbf{Q}_{i}^{1 / 2}\right.\right. \\
& \left.\left.\quad+\mathbf{I}_{n_{s}}\right)^{-1} \boldsymbol{\Theta}_{i}^{-1 / 2} \overline{\mathbf{H}}_{j} \mathbf{R}_{x_{j}}\right] .
\end{aligned}
$$

Observing that only the term $p_{i}(\mathbf{F})$ is related to $\mathbf{F}$, the optimization problem in (14) is equivalent to minimizing $p_{1}(\mathbf{F})+p_{2}(\mathbf{F})$. Assuming $p_{i}(\mathbf{F}) \leq \bar{p}_{i}(\mathbf{F})$ by contraction method, where

$$
\begin{aligned}
& \bar{p}_{i}(\mathbf{F}) \\
& \quad=\rho_{j} \operatorname{Tr}\left[\boldsymbol { \Theta } _ { i } ^ { - 1 / 2 } \overline { \mathbf { H } } _ { j } \mathbf { R } _ { x _ { j } } ^ { 2 } \overline { \mathbf { H } } _ { j } ^ { H } \boldsymbol { \Theta } _ { i } ^ { - 1 / 2 } \left(\rho_{i} \boldsymbol{\Theta}_{i}^{1 / 2} \mathbf{F}^{H} \overline{\mathbf{H}}_{i} \overline{\boldsymbol{\Phi}}_{i}^{-1} \overline{\mathbf{H}}_{i}^{H} \mathbf{F} \Theta_{i}^{1 / 2}\right.\right. \\
& \left.\left.\quad+\mathbf{I}_{n_{s}}\right)^{-1}\right]
\end{aligned}
$$

and here $\overline{\boldsymbol{\Phi}}_{i}=P_{r} \mathbf{Z}_{i}+\mathbf{R}_{n_{i}}$, hence the original optimization problem (14) can be simplified as

$$
\begin{array}{ll}
\min _{\mathbf{F}} & \mathrm{MSE}=\bar{p}_{1}(\mathbf{F})+\bar{p}_{2}(\mathbf{F}) \\
\text { s.t. } & \operatorname{Tr}\left(\mathbf{F R}_{r} \mathbf{F}^{H}\right) \leq P_{r} .
\end{array}
$$

Next, using matrix decomposition method to simplify the structure of $\bar{p}_{i}(\mathbf{F})$, it can be rewritten as

$$
\bar{p}_{i}(\mathbf{F})=\operatorname{Tr}\left[\mathbf{X}_{i}\left(\Theta_{i}^{1 / 2} \mathbf{F}^{H} \boldsymbol{\Xi}_{i} \mathbf{F} \Theta_{i}^{1 / 2}+\mathbf{I}_{n_{s}}\right)^{-1}\right]
$$

and here $\boldsymbol{\Xi}=\overline{\mathbf{H}}_{i}^{H} \overline{\boldsymbol{\Phi}}_{i}^{-1} \overline{\mathbf{H}}_{i}$ and $\mathbf{X}_{i}=\boldsymbol{\Theta}_{i}^{-1 / 2} \overline{\mathbf{H}}_{j} \mathbf{R}_{x_{j}}^{2} \overline{\mathbf{H}}_{j}^{H} \boldsymbol{\Theta}_{i}^{-1 / 2} . \mathbf{X}_{i}$ can be expressed as $\mathbf{X}_{i}=\mathbf{U}_{X_{i}} \Lambda_{X_{i}} \mathbf{U}_{X_{i}}^{H}$ by EVD decomposition, $\mathbf{U}_{X_{i}}$ is unitary matrix, and $\Lambda_{X_{i}}$ is diagonal matrix. Expression (21) can be described as

$$
\begin{aligned}
& \bar{p}_{i}(\mathbf{F}) \\
& =\rho_{j} \operatorname{Tr}\left[\boldsymbol{\Lambda}_{X_{i}} \mathbf{U}_{X_{i}}^{H}\left(\boldsymbol{\Theta}_{i}^{1 / 2} \mathbf{F}^{H} \mathbf{\Xi}_{i}^{H / 2} \boldsymbol{\Xi}_{i}^{1 / 2} \mathbf{F} \boldsymbol{\Theta}_{i}^{1 / 2}+\mathbf{I}_{n_{s}}\right)^{-1}\right. \\
& \left.\cdot \mathbf{U}_{X_{i}}\right]=\rho_{j} \operatorname{Tr}\left[\boldsymbol{\Lambda}_{X_{i}}\left(\mathbf{Y}_{i}^{H} \mathbf{F}^{H} \mathbf{\Xi}_{i}^{H / 2} \mathbf{\Xi}_{i}^{1 / 2} \mathbf{F} \mathbf{Y}_{i}+\mathbf{I}_{n_{s}}\right)^{-1}\right]
\end{aligned}
$$

and here $\mathbf{Y}_{i}=\boldsymbol{\Theta}_{i}^{1 / 2} \mathbf{U}_{X_{i}}$. We propose the following structure:

$$
\mathbf{F}=\mathbf{U}_{\Xi} \boldsymbol{\Lambda}_{F} \mathbf{U}_{Y}^{-1}
$$

Here, $\mathbf{U}_{\Xi}$ is unitary, $\boldsymbol{\Lambda}_{F}$ is $n_{r} \times n_{r}$ diagonal, and $\mathbf{U}_{Y}$ is the nonsingular matrix obtained by the generalized singular value decomposition (GSVD) from $\mathbf{Y}_{1}$ and $\mathbf{Y}_{2} \cdot \mathbf{Y}_{i}^{H}$ can be written as

$$
\mathbf{Y}_{i}^{H}=\mathbf{V}_{Y_{i}} \Lambda_{Y_{i}} \mathbf{U}_{Y}^{H}
$$

where $\mathbf{V}_{Y_{i}}$ is unitary and $\Lambda_{Y_{i}}$ is diagonal. $\mathbf{U}_{\Xi}^{H}$ is obtained from the following singular value decomposition (SVD):

$$
\Xi_{i}^{1 / 2}=\left(V_{\Xi_{i}} \widetilde{V}_{\Xi_{i}}\right)\left(\begin{array}{c}
\Lambda_{\Xi} \\
0
\end{array}\right) U_{\Xi}^{\mathrm{H}}
$$

and here $\Lambda_{\Xi}$ is $n_{r} \times n_{r}$ diagonal, from (25), and $\boldsymbol{\Xi}_{i}^{1 / 2}=$ $\mathbf{V}_{\Xi_{i}} \boldsymbol{\Lambda}_{\Xi} \mathbf{U}_{\Xi}^{H}$. Substituting (23) (25) into (22) gives

$$
\bar{p}_{i}(\mathbf{F})=\rho_{j} \operatorname{Tr}\left[\mathbf{A}_{i}\left(\rho_{i} \mathbf{V}_{\Xi_{i}}^{H} \mathbf{V}_{\Xi_{i}} \Lambda_{\Xi}^{2} \Lambda_{F}^{2} \Lambda_{Y_{i}}^{2}+\mathbf{I}_{n_{s}}\right)^{-1}\right]
$$

and here $\mathbf{A}_{i}=\mathbf{V}_{Y_{i}}^{H} \boldsymbol{\Lambda}_{X_{i}} \mathbf{V}_{Y_{i}}$. In order to achieve some simple solutions, we further relax the problem by only considering the main diagonal of $\mathbf{V}_{\Xi_{i}}^{H} \mathbf{V}_{\Xi_{i}}$. Finally, the problem in (20) is simplified to the following problem:

$$
\begin{array}{ll}
\min _{\boldsymbol{\Lambda}_{F}} & \rho_{j} \operatorname{Tr}\left[\mathbf{A}_{i}\left(\mathbf{I}_{n_{s}}+\mathbf{U}_{\Xi_{i}} \boldsymbol{\Lambda}_{\Xi}^{2} \boldsymbol{\Lambda}_{B_{i}}^{2} \boldsymbol{\Lambda}_{F}^{2}\right)^{-1}\right] \\
\text { s.t. } & \operatorname{Tr}\left(\mathbf{D} \boldsymbol{\Lambda}_{F}^{2}\right) \leq P_{r},
\end{array}
$$

where $\mathbf{U}_{\Xi_{i}}=\operatorname{diag}\left(\mathbf{V}_{\Xi_{i}}^{H} \mathbf{V}_{\Xi_{i}}\right)$ and $\mathbf{D}=\mathbf{U}_{B}^{-1} \mathbf{R}_{r} \mathbf{U}_{B}^{-H}$; the optimization problem (27) can be rewritten in scalar form as

$$
\begin{aligned}
\min _{\eta_{1}, \eta_{2}, \ldots, \eta_{n_{s}}} & \sum_{i=1}^{2} \sum_{n=1}^{n_{s}} \frac{\rho_{i} \rho_{j} \Phi_{i n}}{1+\mu_{i n} \eta_{n}} \\
\text { s.t. } & \sum_{n=1}^{n_{s}} d_{n} \eta_{n} \leq P_{r},
\end{aligned}
$$

where $\eta_{n}, \mu_{i n}, \omega_{i n}$, and $d_{n}$ are the diagonal entries of $\Lambda_{F}^{2}$, $\mathbf{U}_{\Xi_{i}} \Lambda_{\Xi}^{2} \Lambda_{B_{i}}^{2}, \mathbf{A}_{i}$, and $\mathbf{D}$. It is easy to recognize that (28) is a convex optimization problem $[24,25]$; translating the nonconvex problem of formula (28) into a convex optimization problem by Lagrange multiplier method, $\lambda$ is the Lagrange multiplier, and the Lagrange function is constructed for

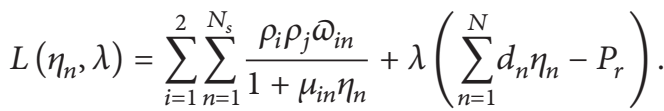

$\partial L / \partial \eta_{n}=0$ gives

$\eta_{n}$

$$
=\frac{-2 \lambda d_{n} \mu_{i n}+\sqrt{4 \lambda^{2} d_{n}^{2} \mu_{i n}^{2}-4 \lambda d_{n} \mu_{i n}^{2}\left(\lambda d_{n}-\sum_{i=1}^{2} \rho_{i} \rho_{j} \mu_{i n} \Phi_{i n}\right)}}{2 \lambda d_{n} \mu_{i n}^{2}}
$$

$$
=-\frac{1}{\sum_{i=1}^{2} \mu_{i n}}+\sqrt{\sum_{i=1}^{2} \frac{\rho_{i} \rho_{j} \omega_{i n}}{\lambda d_{n} \mu_{i n}}} .
$$




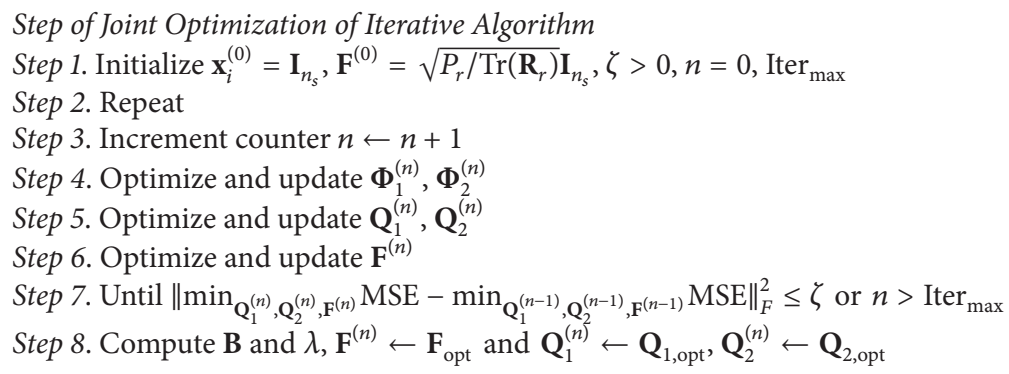

Algorithm 1: Joint optimization of iterative algorithm.

$\lambda$ is in accord with

$$
\begin{aligned}
\lambda & =\sum_{i=1}^{2} \frac{\rho_{i} \rho_{j} \mu_{i n} \Phi_{i n}}{d_{n}\left(1+\mu_{i n} \eta_{n}\right)^{2}} \leq \sum_{i=1}^{2} \frac{\rho_{i} \rho_{j} \mu_{i n} \Phi_{i n}}{d_{n}} \\
& \leq \max \left\{\sum_{i=1}^{2} \frac{\rho_{i} \rho_{j} \mu_{i n} \Phi_{i n}}{d_{n}}, \forall=1,2, \ldots, n_{s}\right\}
\end{aligned}
$$

and then

$$
0 \leq \lambda \leq \max \left\{\sum_{i=1}^{2} \frac{\rho_{i} \rho_{j} \mu_{i n} \Phi_{i n}}{d_{n}}, \forall=1,2, \ldots, n_{s}\right\} .
$$

$\lambda$ should be chosen from bisection algorithm and satisfied with $\sum_{n=1}^{n_{s}} d_{n} \eta_{n}=P_{r}, \eta_{n}$ could be obtained by substituting $\lambda$ into (30), and then $\Lambda_{F}^{2}, \mathbf{F}, \mathbf{Q}_{1}$, and $\mathbf{Q}_{2}$ will be gotten as follows.

4.2. Joint Iterative Algorithm Design. When the detection matrix $\mathbf{Q}_{i}$ is determined, the optimization problem of (14) can be translated into a convex optimization problem of relay precoding matrix $\mathbf{F}$. The Lagrangian function of (14) is formulated as

$$
L(\mathbf{F}, \lambda)=\min _{\mathbf{Q}_{1}, \mathbf{Q}_{2}, \mathbf{F}} \mathrm{MSE}+\lambda\left[\operatorname{Tr}\left(\mathbf{F R}_{r} \mathbf{F}^{H}\right)-P_{r}\right] .
$$

Seeking partial derivative of $\mathbf{F}^{*}$

$$
\begin{aligned}
\left.\frac{\partial L}{\partial \mathbf{F}}\right|_{\mathbf{F}=\mathbf{F}^{*}}= & \sum_{i=1}^{2} \rho_{i} \overline{\mathbf{H}}_{i} \mathbf{Q}_{i}^{H} \mathbf{Q}_{i} \overline{\mathbf{H}}_{i}^{H} \mathbf{F} \Theta_{i} \\
& +\sum_{i=1}^{2} \operatorname{Tr}\left(\mathbf{Q}_{i} \mathbf{Z}_{i} \mathbf{Q}_{i}^{H}\right) \mathbf{F} \boldsymbol{\Omega}_{j} \\
& -\sum_{i=1}^{2} \sqrt{\rho_{i} \rho_{j}} \overline{\mathbf{H}}_{i} \mathbf{Q}_{i}^{H} \mathbf{R}_{x_{j}} \overline{\mathbf{H}}_{j}^{H}+\lambda \mathbf{F} \mathbf{R}_{r},
\end{aligned}
$$

where $\boldsymbol{\Omega}_{j}=\boldsymbol{\Pi}_{j}+\overline{\mathbf{H}}_{i} \mathbf{R}_{x_{i}} \overline{\mathbf{H}}_{i}^{H}+\mathbf{R}_{n_{r}}$; together with the complementary slackness and the relay station transmit power constraint, we obtain the KKT conditions as

$$
\begin{aligned}
\left.\frac{\partial L}{\partial \mathbf{F}}\right|_{\mathbf{F}=\mathbf{F}^{*}} & =0, \\
\lambda\left[\operatorname{Tr}\left(\mathbf{F R}_{r} \mathbf{F}^{H}\right)-P_{r}\right] & =0, \\
\operatorname{Tr}\left(\mathbf{F R}_{r} \mathbf{F}^{H}\right) & \leq P_{r}
\end{aligned}
$$

and from (34) and (35) one has

$$
\operatorname{vec}(\mathbf{F})=\Gamma^{-1} \operatorname{vec}(\mathbf{Q}),
$$

where

$$
\begin{aligned}
\boldsymbol{\Gamma}= & \sum_{i=1}^{2} \boldsymbol{\Theta}_{i}^{T} \otimes\left(\rho_{i} \overline{\mathbf{H}}_{i} \mathbf{Q}_{i}^{H} \mathbf{Q}_{i} \overline{\mathbf{H}}_{i}^{H}\right) \\
& +\sum_{i=1}^{2} \boldsymbol{\Omega}_{j}^{T} \otimes \operatorname{Tr}\left(\mathbf{Q}_{i} \mathbf{Z}_{i} \mathbf{Q}_{i}^{H}\right)+\lambda \mathbf{R}_{r}^{T} \otimes \mathbf{I}_{n_{s}}, \\
\mathbf{B}= & \sum_{i=1}^{2} \sqrt{\rho_{i} \rho_{j}} \overline{\mathbf{H}}_{i} \mathbf{Q}_{i}^{H} \mathbf{R}_{x_{j}} \overline{\mathbf{H}}_{j}^{H} .
\end{aligned}
$$

The Lagrange multiplier is determined by (36) and (37). It satisfied [26]

$$
\begin{aligned}
0 & \leq \lambda \\
& \leq \operatorname{Tr}\left[\left(\mathbf{R}_{r}^{T} \otimes \mathbf{I}_{n_{s}}\right)^{-1}\right] \sqrt{\frac{\lambda_{1}\left(\operatorname{vec}(\mathbf{B}) \operatorname{vec}^{H}(\mathbf{B})\right) \lambda_{1}\left(\mathbf{R}_{r}\right)}{P_{r}}}
\end{aligned}
$$

and here $\otimes$ denotes Kronecker product, $\lambda_{1}(\mathbf{A})$ represents the maximum eigenvalue of the matrix $\mathbf{A}$, and $\lambda$ can be obtained by bisection algorithm with its upper and lower bounds. By (17) and (38), we can see that when the signal matrix $\mathbf{x}$ of the source node is certain, the relay matrix and the detection matrix are correlated. A joint iterative design algorithm is proposed as shown in Algorithm 1.

\section{Simulation Results and Analysis}

In this section, simulation results are provided to evaluate the performance of the proposed approaches in a flat Rayleigh fading environment. We consider that the source nodes $S_{1}$ and $S_{2}$ are equipped with $n_{s}=4$ antennas. Relay node $F$ is equipped with $n_{r}=4$ antennas. The source node transmits independent uncoded QPSK symbol streams to the corresponding receiver. The covariance of the transmit signal at source nodes $S_{1}$ and $S_{2}$ is $\mathbf{R}_{x_{1}}=\mathbf{R}_{x_{2}}=\left(P_{s} / n_{s}\right) \mathbf{I}_{n_{s}}$, and here $P_{s}$ is the transmit power at each source node; let $P_{s}=n_{s}$. The covariance of the AWGN is satisfied with $\mathbf{R}_{n_{r}}=\sigma_{r}^{2} \mathbf{I}_{n_{r}}$. and $\mathbf{R}_{n_{1}}=\mathbf{R}_{n_{2}}=\sigma_{s}^{2} \mathbf{I}_{n_{r}}$. We investigate the scenarios with 


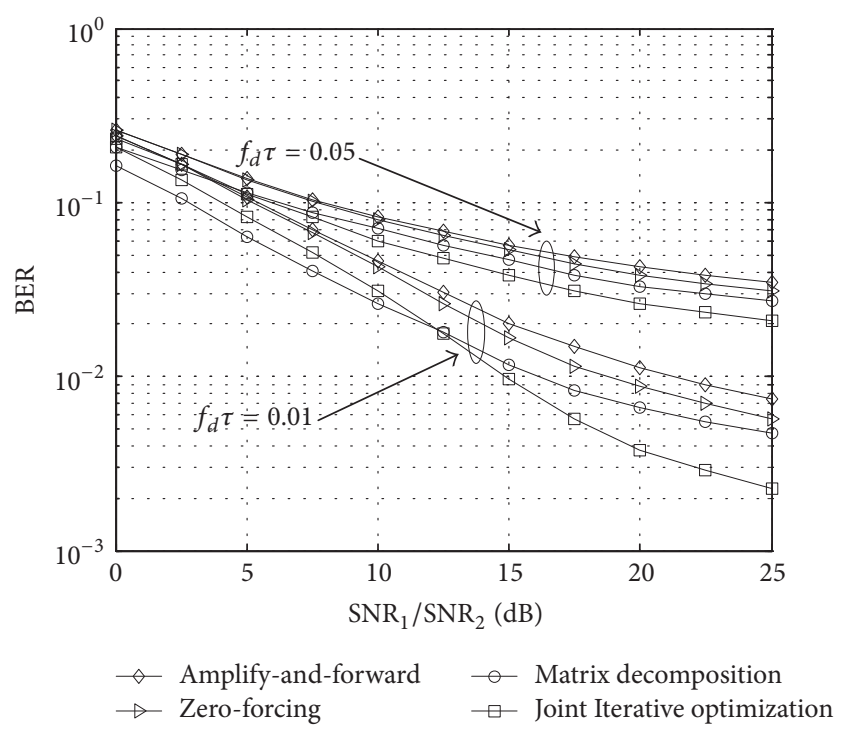

FIGURE 2: BER versus SNR with $\sigma_{\Sigma}^{2}=0.01$.

various $\mathrm{SNR}_{1}=P_{s} /\left(n_{s} \sigma_{s}^{2}\right)$ while fixing $\mathrm{SNR}_{2}=P_{r} /\left(n_{r} \sigma_{r}^{2}\right)$. $\mathrm{SNR}_{1}$ and $\mathrm{SNR}_{2}$ are the average signal-to-noise ratio (SNR) for the first phase and the second phase of the two-way communication protocol. The results are averaged over 5000 independent channel realizations, and in each realization 2000 QPSK symbols are transmitted for each data stream. In order to verify the superiority of the proposed algorithm, the proposed methods are compared with the existing methods as follows:

(i) Amplify-and-forward (AF) relay approach [27] is

$$
\mathbf{F}_{\mathrm{AF}} \propto \sqrt{\frac{P_{r}}{\operatorname{Tr}\left(\mathbf{R}_{r}\right)}} \mathbf{I}_{n_{r}} .
$$

(ii) Zero-forcing (ZF) relay approach [28] is

$$
\begin{aligned}
\mathbf{F}_{\mathrm{ZF}}= & \left(\sum_{i=1}^{2} \rho_{i} \overline{\mathbf{H}}_{i} \mathbf{Q}_{i}^{H} \mathbf{Q}_{i} \overline{\mathbf{H}}_{i}^{H} \mathbf{\Theta}_{i}+\lambda \mathbf{R}_{r}\right)^{-1} \\
& \cdot\left(\sum_{i=1}^{2} \sqrt{\rho_{i} \rho_{j}} \overline{\mathbf{H}}_{i} \mathbf{Q}_{i}^{H} \mathbf{R}_{x_{j}} \overline{\mathbf{H}}_{j}^{H}\right), \quad \mathbf{Q}_{i} \propto \mathbf{I}_{n_{r}} .
\end{aligned}
$$

Figures 2 and 3 are given the performance comparison of BER while fixing estimated errors $\sigma_{\Sigma}^{2}=\sigma_{\Sigma_{1}}^{2}=\sigma_{\Sigma_{2}}^{2}$ and delay coefficients $f_{d} \tau_{1}=f_{d} \tau_{2}=f_{d} \tau$. Simulation parameters are as follows: iterations Iter ${ }_{\max }=30$ and convergence threshold $\zeta=10^{-4}$.

Figure 4 is given the performance comparison of BER with different iteration numbers. Simulation parameters are as follows: $\sigma_{\Sigma}^{2}=0.002, f_{d} \tau=0.02$, and convergence threshold $\zeta=10^{-4}$.

Figures 5 and 6 are the BER performance comparisons in the two kinds of SNR while fixing feedback delay coefficient $f_{d} \tau=0.02$ and estimation error variance $\sigma_{\Sigma}^{2}=0.02$, respectively. The simulation parameters are as follows: iterations Iter $_{\max }=30$ and convergence threshold $\zeta=10^{-4}$.

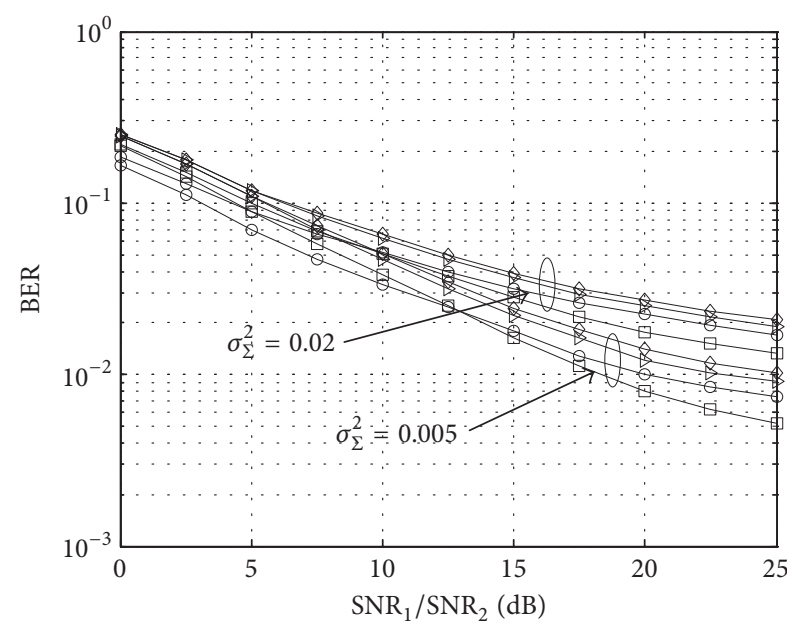

$$
\begin{array}{lll}
\diamond \text { Amplify-and-forward } & - & \text { Matrix decomposition } \\
\rightarrow \text { Zero-forcing } & \square & \text { Joint Iterative optimization }
\end{array}
$$

FIgURE 3: BER versus SNR with $f_{d} \tau=0.05$.

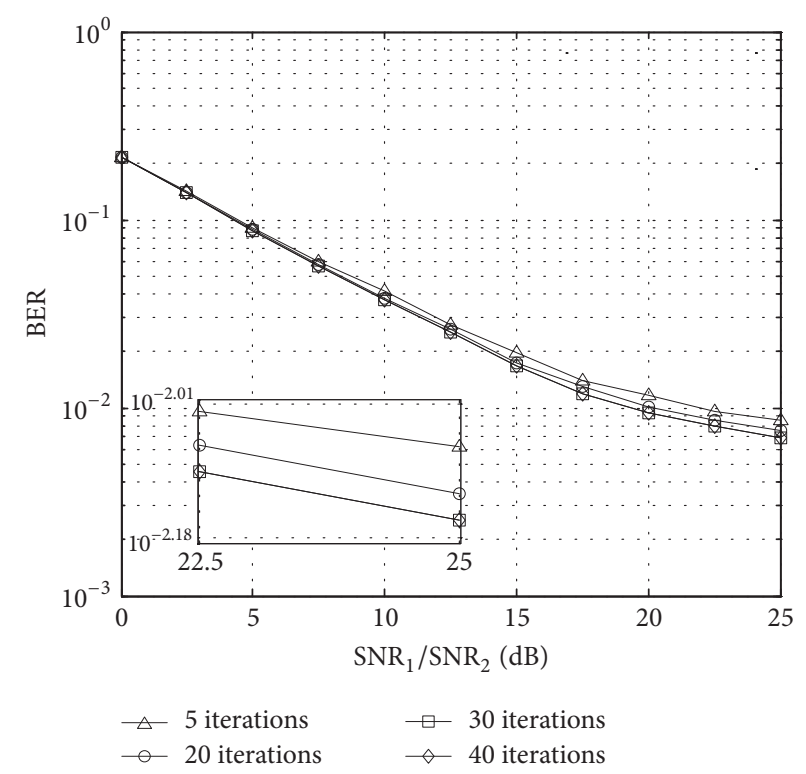

FIGURE 4: BER performance comparisons with different iterations.

From the above simulations, the following conclusions can be drawn:

(1) From Figures 2 and 3, the joint iterative algorithm achieved $3.2 \mathrm{~dB}$ and $4 \mathrm{~dB}$ performance gain compared to the ZF scheme and AF scheme while fixing $f_{d} \tau=$ $0.05, \sigma_{\Sigma}^{2}=0.005$, and BER $=0.05$, respectively. The matrix decomposition algorithm could obtain $2 \mathrm{~dB}$ and $3 \mathrm{~dB}$ performance gain compared to the $\mathrm{ZF}$ scheme and AF scheme. And it is obvious that matrix decomposition algorithm could get the optimal system BER performance when the channel estimation error and feedback delay coefficient are certain in low SNR conditions. As the SNR increases to $10 \mathrm{~dB}$, the joint iterative algorithm obtains the best BER 


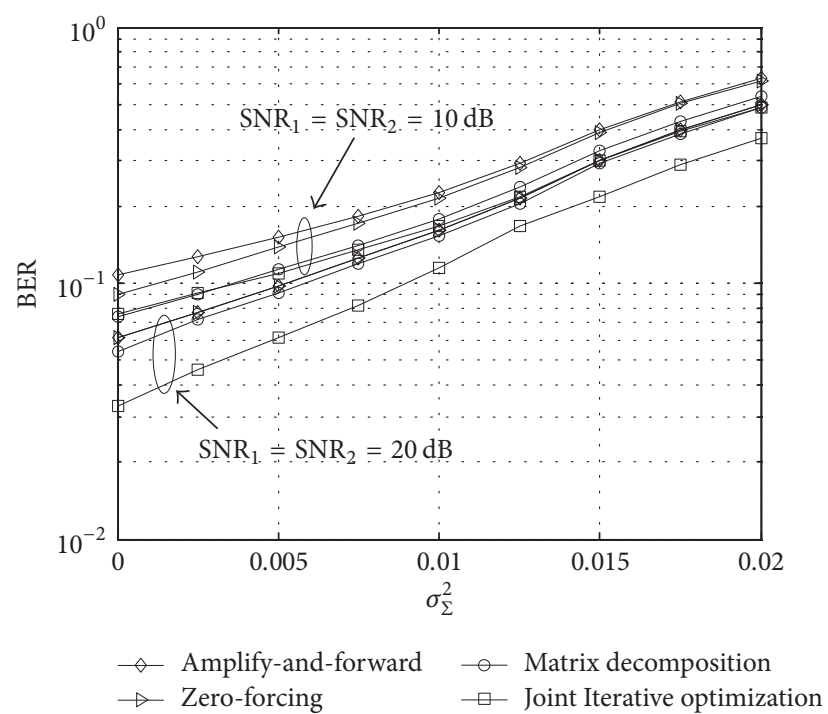

FIGURE 5: BER performance comparisons with different $\sigma_{\Sigma}^{2}$.

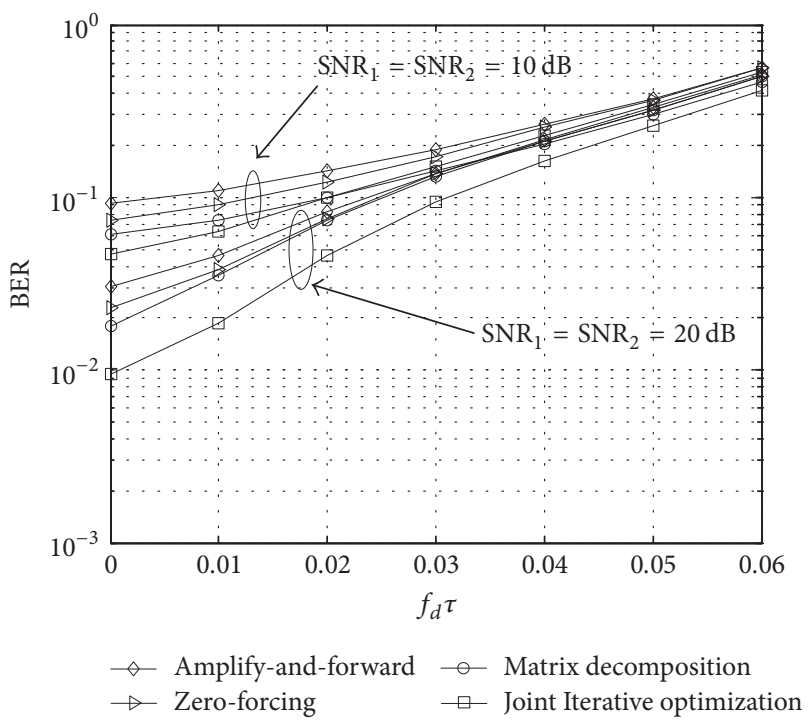

FIGURE 6: BER performance comparisons with different $f_{d} \tau$.

performance. Compared to the AF relay approach and $\mathrm{ZF}$ relay approach, the proposed two schemes have obvious advantages. Simulation results show that the proposed algorithm significantly improves the BER performance compared with the existing approaches. It is because the used AF relay scheme only amplifies and forwards the signal, while the existing ZF scheme ignores the interference signal of the relay node and performs the weighting processing on signal only. Moreover, the proposed method not only considers the channel estimation errors and feedback delay at each node but also can design each node matrix jointly. Thus, it can obtain obvious performance superiority over the other two methods.

(2) As shown in Figure 4, the system BER performance could be improved by $1-2 \mathrm{~dB}$ with the increase of the number of iterations. However, this increase is limited. When the iteration number is more than 30 times, BER remains almost constant. By defining the threshold value of iterations as 30 , the joint iterative method can obtain the best performance.

(3) From Figures 5 and 6, we find that the system BER performance has a downward trend with the increase of the feedback delay coefficient or the channel estimation error; however, the proposed scheme achieves the best BER performance because it has a certain extent compensated with feedback delay coefficient and estimation error.

\section{Conclusion}

This paper investigates a linear precoding scheme design for the MIMO two-way relay system with imperfect CSI. The transceiver design is simplified as an optimal problem with precoding matrix variables, which is deduced with the maximum power constraint at the relay station based on the MMSE criterion. With channel feedback delay at both ends of the channel and the channel estimation errors being taken into account, a matrix decomposition scheme and a joint iterative scheme are proposed to minimize the BER. Numerical simulation results show that the matrix decomposition scheme can improve the system BER effectively and the joint iterative scheme can achieve the best BER performance with the increase of SNR against existing methods.

\section{Conflicts of Interest}

The authors declared that they have no conflicts of interest to this work. We declare that we do not have any commercial or associative interest that represents conflicts of interest in connection with the work submitted.

\section{Acknowledgments}

This work is jointly supported by the Fundamental Research Funds for the Central Universities (no. NS2016044).

\section{References}

[1] L. Sanguinetti, A. A. D'Amico, and Y. Rong, "A tutorial on the optimization of amplify-and-forward MIMO relay systems," IEEE Journal on Selected Areas in Communications, vol. 30, no. 8, pp. 1331-1346, 2012.

[2] J. Chen, X. Chen, W. H. Gerstacker, and D. W. K. Ng, "Resource allocation for a massive MIMO relay aided secure communication," IEEE Transactions on Information Forensics and Security, vol. 11, no. 8, pp. 1700-1711, 2016.

[3] B. Wang, J. Zhang, and A. Host-Madsen, "On the capacity of MIMO relay channels," IEEE Transactions on Information Theory, vol. 51, no. 1, pp. 29-43, 2005.

[4] D. E. Simmons, J. P. Coon, and N. Warsi, "Capacity and power scaling laws for finite antenna MIMO amplify-and-forward relay networks," IEEE Transactions on Information Theory, vol. 62, no. 4, pp. 1993-2008, 2016. 
[5] J. Yang, B. Champagne, Y. Zou, and L. Hanzo, "Joint optimization of transceiver matrices for MIMO-aided multiuser AF relay networks: improving the QoS in the presence of CSI errors," IEEE Transactions on Vehicular Technology, vol. 65, no. 3, pp. 1434-1451, 2016.

[6] R. Huang, T. Zhang, C. Feng, T. Liang, and F. Li, "Energy efficient power allocation and beamforming in non-regenerative two-way MIMO relay networks," in Proceedings of the International Symposium on Wireless Personal Multimedia Communications (WPMC '14), pp. 65-69, September 2014.

[7] B. Fang, Z. Qian, W. Zhong, and W. Shao, "Joint design for source and relay precoding in AF-based MIMO two-way relay networks," in Proceedings of the IEEE China Summit and International Conference on Signal and Information Processing (ChinaSIP '15), pp. 953-957, IEEE, Chengdu, China, July 2015.

[8] R. Wang and M. Tao, "Joint source and relay precoding designs for MIMO two-way relaying based on MSE criterion," IEEE Transactions on Signal Processing, vol. 60, no. 3, pp. 1352-1365, 2012.

[9] D. Hwang, S. S. Nam, T.-J. Lee, and D. I. Kim, "Finite feedback MIMO precoding for the two-way amplify-and-forward relay network," IEEE Communications Letters, vol. 18, no. 4, pp. 620 623, 2014

[10] W.-C. Choi, S. Choi, A. Heo, and D.-J. Park, "Joint optimization of source and relay for MIMO two-way relay networks using MSE duality," IEEE Communications Letters, vol. 18, no. 7, pp. 1246-1249, 2014.

[11] L. Shen, Y. Fu, C. Liu, and W.-P. Zhu, "Joint relay and destination design for two-way MIMO AF multi-relay systems," in Proceedings of the IEEE 78th Vehicular Technology Conference (VTC Fall '13), pp. 1-5, September 2013.

[12] E. A. Gharavol and E. G. Larsson, "Robust joint optimization of MIMO two-way relay channels with imperfect CSI," in Proceedings of the 49th Annual Allerton Conference on IEEE Communication, Control, and Computing, pp. 1657-1664, 2011.

[13] K. X. Nguyen, Y. Rong, and S. Nordholm, "Simplified MMSE precoding design in interference two-way MIMO relay systems," IEEE Signal Processing Letters, vol. 23, no. 2, pp. 262-266, 2016.

[14] M. Ding and S. D. Blostein, "MIMO minimum total MSE transceiver design with imperfect CSI at both ends," IEEE Transactions on Signal Processing, vol. 57, no. 3, pp. 1141-1150, 2009.

[15] M. A. Hanif and M. H. Lee, "Sum-rate analysis of two-way multi-relay multi-user MIMO networks with two-hop precoding," in Proceedings of the 6th International Conference on Information and Communication Technology Convergence (ICTC '15), pp. 891-893, October 2015.

[16] E. A. Gharavol and E. G. Larsson, "Robust joint optimization of MIMO two-way relay channels with imperfect CSI," in Proceedings of the 49th Annual Allerton Conference on Communication, Control, and Computing (Allerton '11), pp. 1657-1664, IEEE, September 2011.

[17] Y. Rong, M. R. A. Khandaker, and Y. Xiang, "Channel estimation of dual-hop MIMO relay system via parallel factor analysis," IEEE Transactions on Wireless Communications, vol. 11, no. 6, pp. 2224-2233, 2012.

[18] T. M. C. Chu, T. Q. Duong, and H.-J. Zepernick, "Outage probability and ergodic capacity for MIMO-MRT systems under co-channel interference and imperfect CSI," in Proceedings of the IEEE Swedish Communication Technologies Workshop (SweCTW'11), pp. 46-51, October 2011.
[19] T. R. Ramya and S. Bhashyam, "Using delayed feedback for antenna selection in MIMO systems," IEEE Transactions on Wireless Communications, vol. 8, no. 12, pp. 6059-6067, 2009.

[20] E. K. S. Au, S. Jin, M. R. McKay, W. H. Mow, X. Gao, and I. B. Collings, "Analytical performance of MIMO-SVD systems in ricean fading channels with channel estimation error and feedback delay," IEEE Transactions on Wireless Communications, vol. 7, no. 4, pp. 1315-1325, 2008.

[21] H. A. Suraweera, T. A. Tsiftsis, G. K. Karagiannidis, and A. Nallanathan, "Effect of feedback delay on amplify-and-forward relay networks with beamforming," IEEE Transactions on Vehicular Technology, vol. 60, no. 3, pp. 1265-1271, 2011.

[22] C. Xing, Y. Ma, Y. Zhou, and F. Gao, “Transceiver optimization for multi-hop communications with per-antenna power constraints," IEEE Transactions on Signal Processing, vol. 64, no. 6, pp. 1519-1534, 2016.

[23] C. Xing, Y. Jing, and Y. Zhou, "On weighted MSE model for MIMO transceiver optimization," IEEE Transactions on Vehicular Technology, 2017.

[24] S. Xu and Y. Hua, "Optimal design of spatial source-and-relay matrices for a non-regenerative two-way MIMO relay system," IEEE Transactions on Wireless Communications, vol. 10, no. 5, pp. 1645-1655, 2011.

[25] C. Zhao and B. Champagne, "A unified approach to optimal transceiver design for nonregenerative MIMO relaying," IEEE Transactions on Vehicular Technology, vol. 64, no. 7, pp. 29382951, 2015.

[26] C. Xing, S. Li, Z. Fei, and J. Kuan, "How to understand linear minimum mean-square-error transceiver design for multipleinput-multipleoutput systems from quadratic matrix programming," IET Communications, vol. 7, no. 12, pp. 1231-1242, 2013.

[27] C. Song and C. Ling, "On the diversity of linear transceivers in MIMO AF relaying systems," IEEE Transactions on Information Theory, vol. 62, no. 1, pp. 272-289, 2016.

[28] C. Zhai, X. Li, and Y. Hei, "Joint linear transceiver design for uplink multiuser non-regenerative MIMO relay systems," in Proceedings of the 7th International Conference on Wireless Communications, Networking and Mobile Computing (WiCOM '11), pp. 1-4, September 2011. 


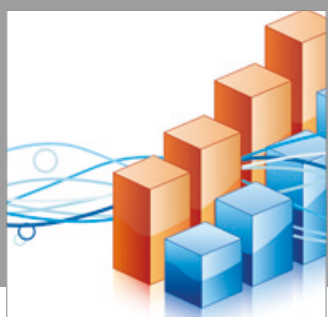

Advances in

Operations Research

vatersals

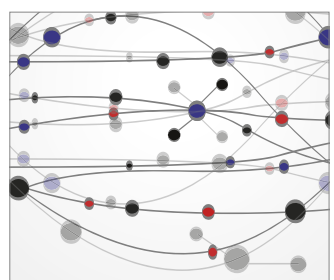

\section{The Scientific} World Journal
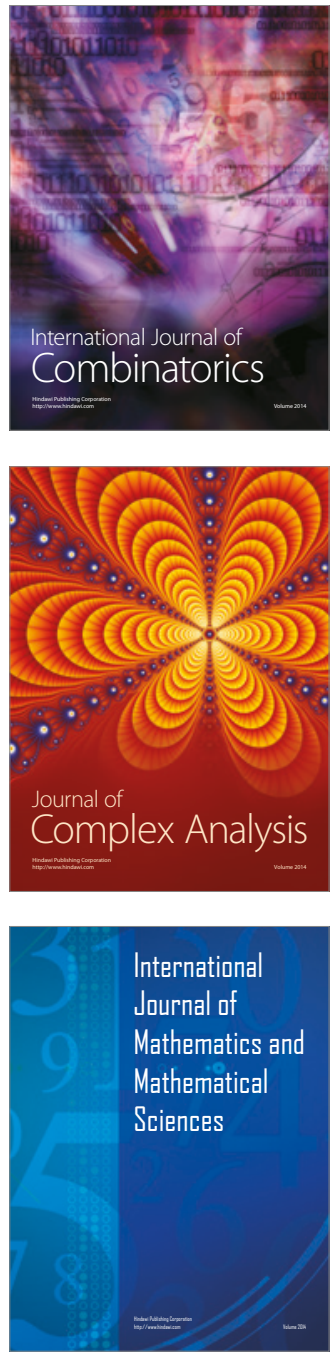
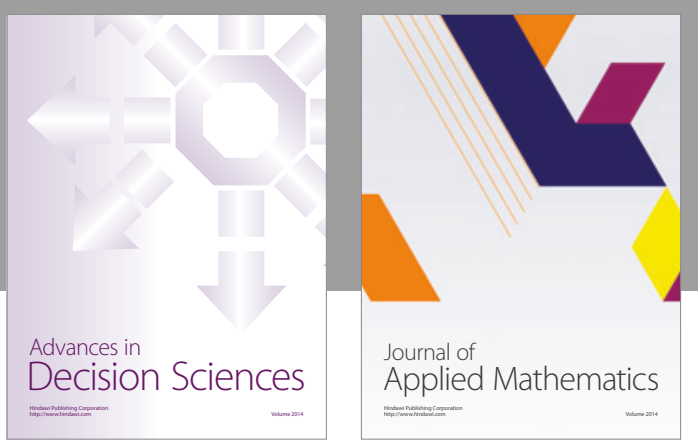

Algebra

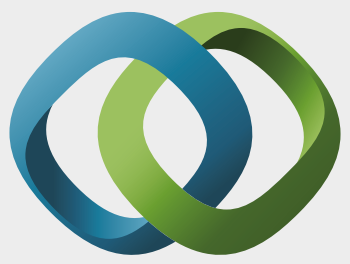

\section{Hindawi}

Submit your manuscripts at

https://www.hindawi.com
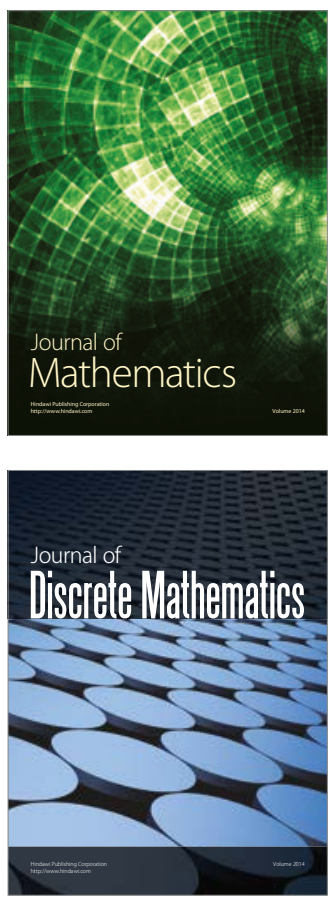

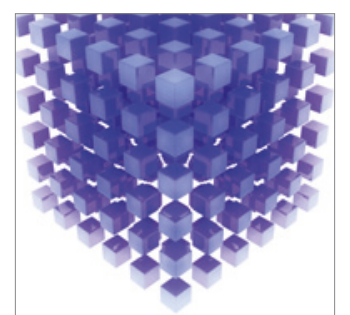

Mathematical Problems in Engineering
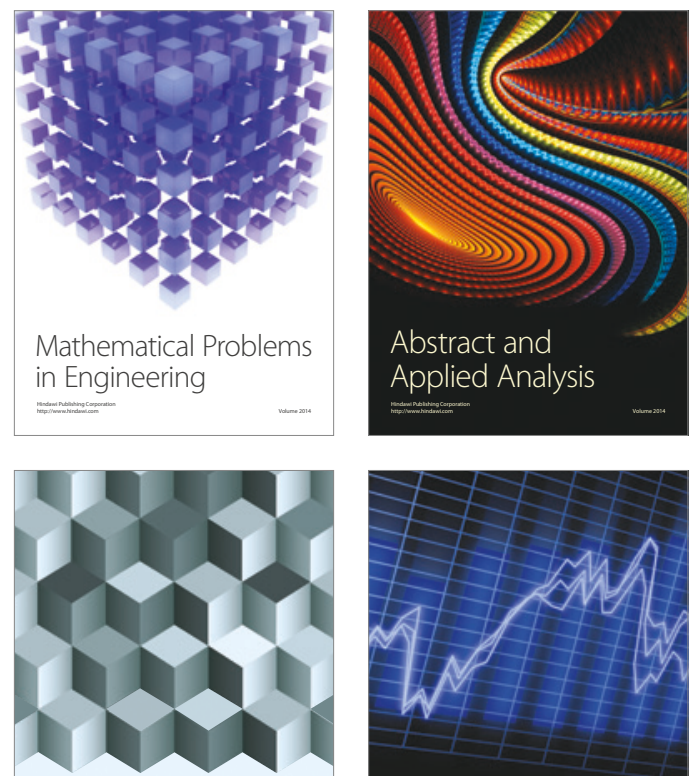

Journal of

Function Spaces

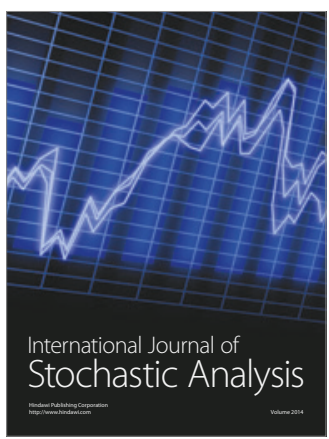

Probability and Statistics
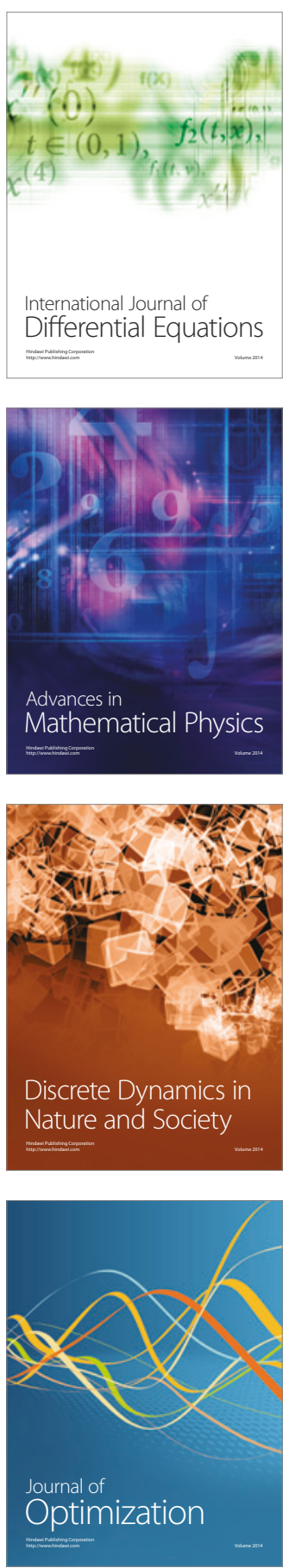